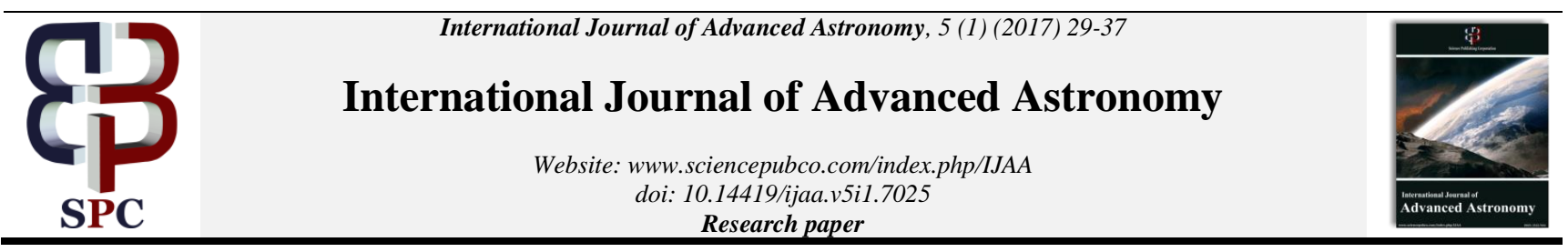

\title{
Pulsating curves of zero velocity for infinitesimal mass around oblate and triaxial rigid body of triangular equilibrium points in elliptical restricted three body problem
}

\author{
Nutan Singh ${ }^{1 *}$, A. Narayan ${ }^{2}$ \\ ${ }^{1}$ Department of Mathematics, Rungta college of Engg\&Tech., Bhilai, 490020Rungta college of Engg.and Technology 491001, India \\ ${ }^{2}$ Department of Mathematics, Bhilai Institute of Technology, Durg, 491001, India \\ *Corresponding author E-mail:ashutoshmaths.narayan@gmail.com
}

\begin{abstract}
This paper explore pulsating Curves of zero velocityof the infinitesimal mass around the triangular equilibrium points with oblate and triaxial rigid body in the elliptical restricted three body problem(ER3BP).
\end{abstract}

Keywords: Er3bp;Triaxiality; Dynamical System; Zero Velocity Curve; Triangular Points.

\section{Introduction}

This paper analyzed the effects of triaxiality and oblateness of both the primaries on the pulsating Curves of zero velocityof the infinitesimal mass moving around the triangular equilibrium points in the elliptical restricted three body problem . It is generally considered that the heavenly bodies are spherical in shape, but actually it has been observed that these bodies are oblate spheroid or triaxial rigid body. There are many planets such as Earth, Jupiter and Saturn which are sufficiently oblate to some degree .Not only this, there are some stars namely Archid, Archerner, Antares, Altairand and Luyten which are either sufficiently oblate or triaxial rigid bodies. The study of these heavenly bodies is significant in study of celestial mechanics and stellar system. The lacks of sphericity of heavenly bodies is one of the reasons of large perturbation. In addition to oblateness of the celestial bodies the triaxiality, atmospheric drag, and solar wind are some other causes of the perturbations.

Many authors such as [1-5] have studied the elliptical restricted three body problem in detail. The influence of eccentricity of orbits of the primaries with or without radiation pressure, oblateness and triaxiality of the primaries are studied by [6-21].

The present study is an attempt to derive the differential equation governing the motion of the triangular equilibrium points on the assumption that both the primaries are oblate triaxial rigid body. The zero velocity curve is plotted by using simulation technique by varying one or more of the parameters.

This paper has five sections, section-1 provides the introduction, section-2 the equation of motion;section-3 describes the location of the triangular equilibrium points;section-4 describes pulsating zero velocity curves; section-5 draws discussion and conclusion.

\section{Equation of motion}

Consider two bodies $S_{1}$ and $S_{2}$ of masses $m_{1}$ and $m_{2}$ with $m_{1} \geq$ $\mathrm{m}_{2}$ which moves in a plane about their common centre of mass $\mathrm{O}$ in Keplerian elliptical orbit having eccentricity e. It is further assumed that the bigger and the smaller primaries having masses $m_{1}$ andm $\mathrm{m}_{2}$ and both are oblate triaxial rigid body. A third body $\mathrm{p}$ of infinitesimal mass moves in the plane of motion $S_{1}$ andS $S_{2}$ under their gravitational attraction without affecting the motion of primaries. The motion of both the primaries affects the motion of the infinitesimal mass. The equation of motion is considered in a fixed co-ordinate system using dimensional quantities and variables .The dimensionless variables has been introduced by using the distance $r$ between the primaries which is given as:

$$
r=\frac{a\left(1-e^{2}\right)}{1+e \cos v}
$$

where a and e are semi-major axis and the eccentricity of the elliptical orbit of the either primary around the other and $v$ is the true anomaly. Suppose $p(x, y)$ are the co-ordinate of the infinitesimal mass of the primaries and the line joining the primaries is taken as $\mathrm{X}$-axis. The $\mathrm{Y}$-axis is taken as the line passing through $\mathrm{O}$ and perpendicular to the $\mathrm{X}$-axis. Let $\mathrm{R}_{1}$ and $\mathrm{R}_{2}$ be the dimensionless distance between the primaries. The equation of motion of the system with the minor modification as solved by $[17,21])$ is given as:

$$
\begin{aligned}
\frac{\mathrm{d}^{2} \mathrm{x}}{\mathrm{dt}^{2}}=-\frac{1}{\mathrm{n}^{2}}\left[\frac{\mathrm{m}_{1} \mathrm{k}^{2}\left(\mathrm{x}-\mathrm{x}_{1}\right)}{\mathrm{R}_{1}{ }^{3}}+\frac{\mathrm{m}_{2} \mathrm{k}^{2}\left(\mathrm{x}-\mathrm{x}_{2}\right)}{\mathrm{R}_{2}{ }^{3}}\right. & \\
& +\frac{3 \mathrm{k}^{2} \mathrm{~m}_{1}\left(\mathrm{x}-\mathrm{x}_{1}\right)\left(2 \sigma_{1}-\sigma_{2}\right)}{2 \mathrm{R}_{1}{ }^{3} \mathrm{r}_{1}{ }^{2}} \\
& -\frac{15 \mathrm{k}^{2} \mathrm{~m}_{1}\left(\mathrm{x}-\mathrm{x}_{1}\right)\left(\sigma_{1}-\sigma_{2}\right) \mathrm{y}^{2}}{2 \mathrm{R}_{1}{ }^{3} \mathrm{r}_{1}{ }^{4}} \\
& +\frac{3 \mathrm{k}^{2} \mathrm{~m}_{2}\left(\mathrm{x}-\mathrm{x}_{2}\right)\left(2 \sigma_{1}{ }^{\prime}-\sigma_{2}{ }^{\prime}\right)}{2 \mathrm{R}_{2}{ }^{3} \mathrm{r}_{2}{ }^{2}} \\
& \left.-\frac{15 \mathrm{k}^{2} \mathrm{~m}_{2}\left(\mathrm{x}-\mathrm{x}_{2}\right)\left(\sigma_{1}{ }^{\prime}-\sigma_{2}{ }^{\prime}\right) \mathrm{y}^{2}}{2 \mathrm{R}_{2}{ }^{3} \mathrm{r}_{2}{ }^{4}}\right]
\end{aligned}
$$




$$
\begin{aligned}
& \frac{\mathrm{d}^{2} \mathrm{y}}{\mathrm{dt}^{2}}=-\frac{1}{\mathrm{n}^{2}}\left[\frac{\mathrm{m}_{1} \mathrm{k}^{2}\left(\mathrm{y}-\mathrm{y}_{1}\right)}{\mathrm{R}_{1}{ }^{3}}+\frac{\mathrm{m}_{2} \mathrm{k}^{2}\left(\mathrm{y}-\mathrm{y}_{2}\right)}{\mathrm{R}_{2}{ }^{3}}+\frac{3 \mathrm{k}^{2} \mathrm{~m}_{1}\left(\mathrm{y}-\mathrm{y}_{1}\right)\left(2 \sigma_{1}-\sigma_{2}\right)}{2 \mathrm{R}_{1}{ }^{3} \mathrm{r}_{1}{ }^{2}}+\right. \\
& \frac{3 \mathrm{k}^{2} \mathrm{~m}_{1}\left(\mathrm{y}-\mathrm{y}_{1}\right)\left(\sigma_{1}-\sigma_{2}\right) \mathrm{q}_{1}}{2 \mathrm{R}_{1}{ }^{3} \mathrm{r}_{1}{ }^{2}}-\frac{15 \mathrm{k}^{2} \mathrm{~m}_{1}\left(\mathrm{y}-\mathrm{y}_{1}\right)\left(\sigma_{1}-\sigma_{2}\right) \mathrm{y}^{2}}{2 \mathrm{R}_{1}{ }^{3} \mathrm{r}_{1}{ }^{4}}- \\
& \frac{15 \mathrm{k}^{2} \mathrm{~m}_{2}\left(\mathrm{y}-\mathrm{y}_{2}\right)\left(\sigma_{1}{ }^{\prime}-\sigma_{2}{ }^{\prime}\right) \mathrm{y}^{2}}{2 \mathrm{R}_{2}{ }^{3} \mathrm{r}_{2}{ }^{4}}+\frac{3 \mathrm{k}^{2} \mathrm{~m}_{2}\left(\mathrm{y}-\mathrm{y}_{2}\right)\left(2 \sigma_{1}{ }^{\prime}-\sigma_{2}{ }^{\prime}\right)}{2 \mathrm{R}_{2}{ }^{3} \mathrm{r}_{2}{ }^{2}}+ \\
& \left.\frac{3 \mathrm{k}^{2} \mathrm{~m}_{2}\left(\mathrm{y}-\mathrm{y}_{2}\right)\left(\sigma_{1}{ }^{\prime}-\sigma_{2}{ }^{\prime}\right)}{\mathrm{R}_{2}{ }^{3} \mathrm{r}_{2}{ }^{2}}\right]
\end{aligned}
$$

where $\sigma_{1}=\left(A_{1}-A_{2}\right) ; \sigma_{2}=\left(A_{2}-A_{3}\right) ; A_{1}=\frac{a^{2}}{5 R^{2}} ; A_{2}=\frac{b^{2}}{5 R^{2}}$ $; \mathrm{A}_{3}=\frac{\mathrm{c}^{2}}{5 \mathrm{R}^{2}}$ anda, $\mathrm{b}, \mathrm{c}$ are the axis of the triaxial rigid body of bigger primary of mass $m_{1}$. whereas $\sigma_{1}^{\prime}=\left(A_{1}^{\prime}-A_{2}^{\prime}\right) ; \sigma_{2}^{\prime}=\left(A_{2}^{\prime}-A_{3}^{\prime}\right)$; $\mathrm{A}_{1}^{\prime}=\frac{\mathrm{a}^{\prime 2}}{5 \mathrm{R}^{\prime 2}} ; \mathrm{A}_{2}^{\prime}=\frac{\mathrm{b}^{2}}{5 \mathrm{R}^{\prime 2}} ; \mathrm{A}_{3}^{\prime}=\frac{\mathrm{c}^{\prime^{2}}}{5 \mathrm{R}^{\prime 2}} \sigma_{2}$ where $\sigma_{1}, \sigma_{2}, \sigma_{1}^{\prime}$ and $\sigma_{2}^{\prime}$ are oblatenessparameter

where $a^{\prime}, b^{\prime}, c^{\prime}$ are the axis of the triaxial rigid body of smaller primary of mass $\mathrm{m}_{2}$, $\mathrm{k}$ the Gaussian constant , $\mathrm{t}^{*}$ is the dimensionless time.

$$
\mathrm{n}^{2}=1+\frac{3}{2}\left(2 \mathrm{~A}_{1}-\mathrm{A}_{2}-\mathrm{A}_{3}\right)+\frac{3}{2}\left(2 \mathrm{~A}_{1}^{\prime}-\mathrm{A}_{2}^{\prime}-\mathrm{A}_{3}^{\prime}\right)
$$

where

$$
R_{1}^{2}=\left(x-x_{1}\right)^{2}+\left(y-y_{1}\right)^{2}
$$

And

$$
\mathrm{R}_{2}^{2}=\left(\mathrm{x}-\mathrm{x}_{2}\right)^{2}+\left(\mathrm{y}-\mathrm{y}_{2}\right)^{2}
$$

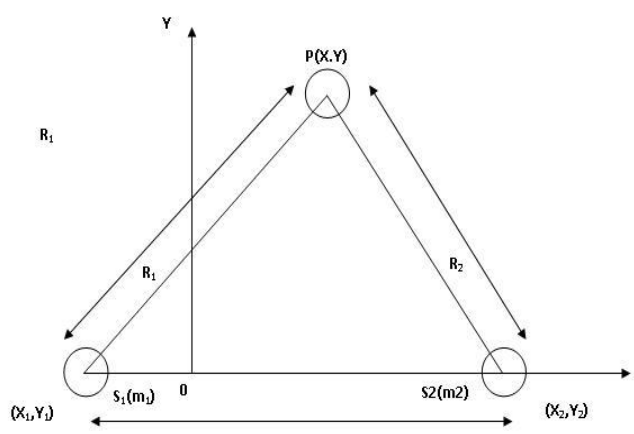

We shall introduce a rotating co-ordinate system $(\bar{x}, \bar{y})$ by substituting

$$
\mathrm{Z}=\overline{\mathrm{z}} \mathrm{e}^{\mathrm{i} v}
$$

where

$$
\mathrm{Z}=\mathrm{X}+\mathrm{iY}
$$

And

$$
\overline{\mathrm{z}}=\overline{\mathrm{x}}+\mathrm{i} \overline{\mathrm{y}}
$$

After introducing the complex vector, the equation of motion (2.1) takes the following form:

$$
\begin{aligned}
& \frac{\mathrm{d}^{2} \mathrm{z}}{\mathrm{dt}^{* 2}}=-\frac{1}{\mathrm{n}^{2}}\left[\frac{\mathrm{m}_{1} \mathrm{k}^{2}\left(\mathrm{z}-\mathrm{z}_{1}\right)}{\mathrm{R}_{1}{ }^{3}}+\frac{\mathrm{m}_{2} \mathrm{k}^{2}\left(\mathrm{z}-\mathrm{z}_{2}\right)}{\mathrm{R}_{2}{ }^{3}}+\frac{3 \mathrm{k}^{2} \mathrm{~m}_{1}\left(\mathrm{z}-\mathrm{z}_{1}\right)\left(2 \sigma_{1}-\sigma_{2}\right)}{2 \mathrm{R}_{1}{ }^{3} \mathrm{r}_{1}{ }^{2}}+\right. \\
& \frac{3 \mathrm{k}^{2} \mathrm{~m}_{2}\left(\mathrm{z}-\mathrm{z}_{2}\right)\left(2 \sigma_{1}{ }^{\prime}-\sigma_{2}{ }^{\prime}\right)}{2 \mathrm{R}_{2}{ }^{3} \mathrm{r}_{2}{ }^{2}}+\frac{3 \mathrm{k}^{2} \mathrm{~m}_{1}\left(\mathrm{z}^{*}-\mathrm{z}_{1}{ }^{3}\right)\left(\sigma_{1}-\sigma_{2}\right)}{\mathrm{R}_{1}{ }^{3} \mathrm{r}_{1}{ }^{2}}+ \\
& \frac{3 \mathrm{k}^{2} \mathrm{~m}_{2}\left(\mathrm{z}^{*}-\mathrm{z}^{*}{ }_{2}\right)\left(\sigma_{1}{ }^{\prime}-\sigma_{2}{ }^{\prime}\right)}{\mathrm{R}_{2}{ }^{3} \mathrm{r}_{2}{ }^{2}}-\frac{15 \mathrm{k}^{2} \mathrm{~m}_{1}\left(\mathrm{z}-\mathrm{z}_{1}\right)\left(\sigma_{1}-\sigma_{2}\right) \mathrm{y}^{2}}{2 \mathrm{R}_{1}{ }^{3} \mathrm{r}_{1}{ }^{4}}- \\
& \left.\frac{15 \mathrm{k}^{2} \mathrm{~m}_{2}\left(\mathrm{z}-\mathrm{z}_{2}\right)\left(\sigma_{1}{ }^{\prime}-\sigma_{2}{ }^{\prime}\right) \mathrm{y}^{2}}{2 \mathrm{R}_{2}{ }^{3} \mathrm{r}_{2}{ }^{4}}\right](2.4)
\end{aligned}
$$

where $\mathrm{z}^{*}=\mathrm{iy}, \mathrm{z}_{1}^{*}=\mathrm{iy}_{1}$ and $\mathrm{z}_{2}^{*}=\mathrm{iy}_{2}$.Now differentiating equation(2.3) twice with respect to $t^{*}$ and taking help of (2.4), we get:

$$
\begin{aligned}
& \frac{\mathrm{d}^{2} \mathrm{z}}{\mathrm{dt}^{* 2}}+2 \mathrm{i} \frac{\mathrm{d} v}{\mathrm{dt}^{*}} \cdot \frac{\mathrm{dz}}{\mathrm{dt}^{*}}=-\frac{1}{\mathrm{n}^{2}}\left[\frac{\mathrm{m}_{1} \mathrm{k}^{2}\left(\mathrm{z}-\mathrm{z}_{1}\right)}{\mathrm{R}_{1}{ }^{3}}+\frac{\mathrm{m}_{2} \mathrm{k}^{2}\left(\mathrm{z}-\mathrm{z}_{2}\right)}{\mathrm{R}_{2}{ }^{3}}+\right. \\
& \frac{3 \mathrm{k}^{2} \mathrm{~m}_{1}\left(\mathrm{z}-\mathrm{z}_{1}\right)\left(2 \sigma_{1}-\sigma_{2}\right)}{2 \mathrm{R}_{1}{ }^{3} \mathrm{r}_{1}{ }^{2}}+\frac{3 \mathrm{k}^{2} \mathrm{~m}_{2}\left(\mathrm{z}-\mathrm{z}_{2}\right)\left(2 \sigma_{1}{ }^{\prime}-\sigma_{2}{ }^{\prime}\right)}{2 \mathrm{R}_{2}{ }^{3} \mathrm{r}_{2}{ }^{2}}+ \\
& \frac{3 \mathrm{k}^{2} \mathrm{~m}_{1}\left(\mathrm{z}^{*}-\mathrm{z}^{*}\right)\left(\sigma_{1}-\sigma_{2}\right)}{\mathrm{R}_{1}{ }^{3} \mathrm{r}_{1}{ }^{2}}+\frac{3 \mathrm{k}^{2} \mathrm{~m}_{2}\left(\mathrm{z}^{*}-\mathrm{z}^{*}{ }_{2}\right)\left(\sigma_{1}{ }^{\prime}-\sigma_{2}{ }^{\prime}\right)}{\mathrm{R}_{2}{ }^{3} \mathrm{r}_{2}{ }^{2}}- \\
& \left.\frac{15 \mathrm{k}^{2} \mathrm{~m}_{1}\left(\mathrm{z}-\mathrm{z}_{1}\right)\left(\sigma_{1}-\sigma_{2}\right) \mathrm{y}^{2}}{2 \mathrm{R}_{1}{ }^{3} \mathrm{r}_{1}{ }^{4}}-\frac{15 \mathrm{k}^{2} \mathrm{~m}_{2}\left(\mathrm{z}-\mathrm{z}_{2}\right)\left(\sigma_{1}{ }^{\prime}-\sigma_{2}{ }^{\prime}\right) \mathrm{y}^{2}}{2 \mathrm{R}_{2}{ }^{3} \mathrm{r}_{2}{ }^{4}}\right]+\mathrm{z}\left(\frac{\mathrm{d} v}{\mathrm{dt}^{*}}\right)^{2}- \\
& \mathrm{iz} \frac{\mathrm{d}^{2} v}{\mathrm{dt}^{* 2}}
\end{aligned}
$$

In equation (2.5), the second term in the left hand side represents the Coriolisacceleration. The complex position vectors $\mathrm{z}_{1}$ and $\mathrm{z}_{2}$ are the location of the primaries, which are permanent on the real axis $(\bar{x}, \bar{y})$ of the system, we have:

$$
\mathrm{z}_{1}=\overline{\mathrm{x}_{1}}=\left(\frac{-\mathrm{p}_{1}}{1+\mathrm{e} \cos v}\right), \mathrm{z}_{2}=\overline{\mathrm{x}_{2}}=\left(\frac{\mathrm{p}_{2}}{1+\mathrm{e} \cos v}\right)
$$

where $\mathrm{p}_{1}$ and $\mathrm{p}_{2}$ are positive and

$$
\frac{\mathrm{p}_{1}}{\mathrm{p}_{2}}=\frac{\mathrm{a}_{1}}{\mathrm{a}_{2}}=\frac{\mathrm{m}_{2}}{\mathrm{~m}_{1}}
$$

where $\mathrm{a}_{1}$ and $\mathrm{a}_{2}$ are the semi major axis of the elliptical orbits of the massive primaries described about their centre of mass, and $\mathrm{m}_{1}, \mathrm{~m}_{2}$ are the masses of the respective bigger and smaller primaries. Further introducing a dimensionless pulsating coordinate system given by:

$$
\rho=\left(\frac{z}{r}\right)=x+i y
$$

where

$$
\mathrm{r}=\frac{\mathrm{a}\left(1-\mathrm{e}^{2}\right)}{1+\mathrm{ecos} v}
$$

Is the distance between the primaries in which a is a semi-major axis of the one primary around other? From equation (2.8) and (2.9), we get:

$$
\begin{aligned}
& X=\frac{\bar{x}(1+e \cos v)}{a\left(1-e^{2}\right)} \\
& y=\frac{\bar{y}(1+e \cos v)}{a\left(1-e^{2}\right)}
\end{aligned}
$$

Since these primaries are fixed in the coordinate system, we have form equation (2.6) and (2.10)

$$
\begin{aligned}
& \mathrm{x}_{1}=\frac{\overline{\mathrm{x}_{1}}(1+\mathrm{e} \cos v)}{\mathrm{a}\left(1-\mathrm{e}^{2}\right)}=\frac{-\mathrm{p}_{1}}{\mathrm{a}\left(1-\mathrm{e}^{2}\right)}=\frac{-\mathrm{a}_{1}}{\mathrm{a}}=-\mu \\
& \mathrm{x}_{2}=\frac{\overline{\mathrm{x}_{2}}(1+\mathrm{ecos} v)}{\mathrm{a}\left(1-\mathrm{e}^{2}\right)}=\frac{\mathrm{p}_{2}}{\mathrm{a}\left(1-\mathrm{e}^{2}\right)}=\frac{\mathrm{a}_{2}}{\mathrm{a}}=1-\mu
\end{aligned}
$$

Here $=\frac{m_{2}}{m_{1}+m_{2}}$. Therefore, the fixed location of these primaries in terms of coordinate $(x, y)$ system are represented as $(-\mu, 0)$ and $(1-\mu, 0)$.The coordinates taken into consideration regarding the problem of two bodies moving in the elliptical orbit are that ,the orbit of $m_{1}$ andm $m_{2}$ with respect to the centre of mass with semimajor axis is $\mathrm{a}_{1}=\mathrm{a} \mu$ and $\mathrm{a}_{2}=\mathrm{a}(1-\mu)$ respectively . In order to solve the equation of motion a true anomaly $v$ is introduced as an independent variable from equation (2.8) we havez $=r \rho$, replacing

$$
\frac{m_{1} k^{2}\left(z-z_{1}\right)}{R_{1}^{3}}=\frac{m_{1} k^{2}\left(\rho-\rho_{1}\right)}{r^{2} r_{1}{ }^{3}}
$$

where

$$
r_{1}^{2}=\left|\rho-\rho_{1}\right|^{2}=(x+\mu)^{2}+y^{2}
$$


Since $\rho_{1}=x_{1}=-\mu$

$$
\frac{m_{2} k^{2}\left(z-z_{2}\right)}{R_{2}{ }^{3}}=\frac{m_{2} k^{2}\left(\rho-\rho_{2}\right)}{r^{2} r_{2}{ }^{3}}
$$

where

$$
r_{2}^{2}=\left|\rho-\rho_{2}\right|^{2}=(x-1+\mu)^{2}+y^{2}
$$

Since

$$
\rho_{2}=x_{2}=1-\mu
$$

And

$$
\frac{3 k^{2} m_{1}\left(z-z_{1}\right)\left(2 \sigma_{1}-\sigma_{2}\right)}{2 R_{1}{ }^{3} r_{1}{ }^{2}}=\frac{3 k^{2} m_{1}\left(\rho-\rho_{1}\right)\left(2 \sigma_{1}-\sigma_{2}\right)}{2 r^{2} r_{1}{ }^{5}}
$$

Similarly

$$
\frac{3 k^{2} m_{2}\left(z-z_{2}\right)\left(2 \sigma_{1}{ }^{\prime}-\sigma_{2}{ }^{\prime}\right)}{2 R_{2}{ }^{3} r_{2}{ }^{2}}=\frac{3 k^{2} m_{2}\left(\rho-\rho_{2}\right)\left(2 \sigma_{1}{ }^{\prime}-\sigma_{2}{ }^{\prime}\right)}{2 r^{2} r_{2}{ }^{5}}
$$

Again

$$
\frac{3 k^{2} m_{1}\left(z^{*}-z_{1}^{*}\right)\left(\sigma_{1}-\sigma_{2}\right)}{R_{1}{ }^{3} r_{1}{ }^{2}}=\frac{3 k^{2} m_{1}\left(\rho^{*}-\rho_{1}^{*}\right)\left(\sigma_{1}-\sigma_{2}\right)}{r^{2} r_{1}{ }^{5}}
$$

Also

$$
\frac{3 k^{2} m_{2}\left(z^{*}-z^{*}{ }_{2}\right)\left(\sigma_{1}{ }^{\prime}-\sigma_{2}{ }^{\prime}\right)}{R_{2}{ }^{3} r_{2}{ }^{2}}=\frac{3 k^{2} m_{2}\left(\rho^{*}-\rho_{2}^{*}\right)\left(\sigma_{1}{ }^{\prime}-\sigma_{2}{ }^{\prime}\right)}{r^{2} r_{2}{ }^{5}}
$$

Similarly

$$
\frac{15 k^{2} m_{1}\left(z-z_{1}\right)\left(\sigma_{1}-\sigma_{2}\right) y^{2}}{2 R_{1}{ }^{3} r_{1}{ }^{4}}=\frac{15 k^{2} m_{1}\left(\rho-\rho_{1}\right)\left(\sigma_{1}-\sigma_{2}\right) y^{2}}{2 r^{2} r_{1}{ }^{7}}
$$

And

$$
\begin{aligned}
& \frac{15 k^{2} m_{2}\left(z-z_{2}\right)\left(\sigma_{1}{ }^{\prime}-\sigma_{2}{ }^{\prime}\right) y^{2}}{2 R_{2}{ }^{3} r_{2}{ }^{4}}= \\
& \frac{15 \boldsymbol{k}^{2} \boldsymbol{m}_{\mathbf{2}}\left(\boldsymbol{\rho}^{*}-\boldsymbol{\rho}^{*}{ }_{2}\right)\left(\boldsymbol{\sigma}_{\mathbf{1}}{ }^{\prime}-\boldsymbol{\sigma}_{\mathbf{2}}{ }^{\prime}\right) \boldsymbol{y}^{\mathbf{2}}}{\mathbf{2} \boldsymbol{r}^{2} \boldsymbol{r}_{\mathbf{2}}{ }^{7}}
\end{aligned}
$$

Now taking $v$ as an independent variable, substituting all required above values in equation (2.5), we get:

$$
\begin{aligned}
& r\left(\frac{d v}{d t^{*}}\right)^{2}\left[\frac{d^{2} \rho}{d v^{2}}+2 i \frac{d \rho}{d v}\right]+\rho\left[\frac{d^{2} r}{d t^{* 2}}-r\left(\frac{d v}{d t^{*}}\right)^{2}\right]+\left(\frac{d \rho}{d v}+i \rho\right) . \\
& {\left[r \frac{d^{2} v}{d t^{* 2}}+2 \frac{d r}{d t^{*}} \frac{d v}{d t^{*}}\right]=-\frac{1}{n^{2}}\left[\frac{m_{1} k^{2}\left(\rho-\rho_{1}\right)}{r^{2} r_{1}{ }^{3}}+\frac{m_{2} k^{2}\left(\rho-\rho_{2}\right)}{r^{2} r_{2}{ }^{3}}+\right.} \\
& \frac{3 k^{2} m_{1}\left(\rho-\rho_{1}\right)\left(2 \sigma_{1}-\sigma_{2}\right)}{2 r^{2} r_{1}{ }^{5}}+\frac{3 k^{2} m_{2}\left(\rho-\rho_{2}\right)\left(2 \sigma_{1}{ }^{\prime}-\sigma_{2}{ }^{\prime}\right)}{2 r^{2} r_{2}{ }^{5}}+ \\
& \frac{3 k^{2} m_{1}\left(\rho^{*}-\rho_{1}^{*}\right)\left(\sigma_{1}-\sigma_{2}\right)}{r^{2} r_{1}{ }^{5}}+\frac{3 k^{2} m_{2}\left(\rho^{*}-\rho^{*}{ }_{2}\right)\left(\sigma_{1}{ }^{\prime}-\sigma_{2}{ }^{\prime}\right)}{r^{2} r_{2}{ }^{5}}- \\
& \left.\frac{15 k^{2} m_{1}\left(\rho-\rho_{1}\right)\left(\sigma_{1}-\sigma_{2}\right) y^{2}}{2 r^{2} r_{1}{ }^{7}}-\frac{15 k^{2} m_{2}\left(\rho-\rho_{2}\right)\left(\sigma_{1}{ }^{\prime}-\sigma_{2}{ }^{\prime}\right) y^{2}}{2 r^{2} r_{2}{ }^{7}}\right](2.21)
\end{aligned}
$$

We have $=r=\left[\frac{a\left(1-e^{2}\right)}{1+e \cos v}\right]$, which is the solution of two body problem involving the primaries $S_{1}$ and $S_{2}$. The integral of angular momentum of two body problem is given by:

$$
\left(r^{2} \frac{d v}{d t^{*}}\right)^{2}=a\left(1-e^{2}\right) k^{2}\left(m_{1}+m_{2}\right)
$$

Differentiating the above equation (2.22), with respect to $t^{*}$, we get:

$$
r \frac{d^{2} v}{d t^{* 2}}+2 \frac{d r}{d t^{*}} \frac{d v}{d t^{*}}=0
$$

The equation of motion ofthe two primaries is given by:

$$
\frac{d^{2} r}{d t^{* 2}}-r\left(\frac{d v}{d t^{*}}\right)^{2}=\frac{-k^{2}\left(m_{1}+m_{2}\right)}{r^{2}}
$$

Using equation (2.22), the equation (2.24) becomes:

$$
\frac{d^{2} r}{d t^{* 2}}-r\left(\frac{d v}{d t^{*}}\right)^{2}=\frac{-1}{a\left(1-e^{2}\right) r^{2}} \cdot\left(\frac{r^{2} d v}{d t^{*}}\right)^{2}
$$

Substituting the value of (2.23), the equation (2.25) becomes

$$
\frac{d^{2} r}{d t^{* 2}}-r\left(\frac{d v}{d t^{*}}\right)^{2}=\frac{-r^{2}}{a\left(1-e^{2}\right)} \cdot\left(\frac{d v}{d t^{*}}\right)^{2}
$$

Substituting the values of (2.23) and (2.26) in equation (2.21) we get

$\left(\frac{d^{2} \rho}{d v^{2}}\right)+2 i \frac{d \rho}{d v}=\frac{r}{a\left(1-e^{2}\right)}\left[\rho-\frac{1}{n^{2}}\left\{\frac{m_{1}}{m_{1}+m_{2}} \frac{\left(\rho-\rho_{1}\right)}{r_{1}^{3}}+\frac{m_{2}}{m_{1}+m_{2}} \frac{\left(\rho-\rho_{2}\right)}{r_{2}^{3}}+\right.\right.$

$\frac{3 m_{1}}{m_{1}+m_{2}} \frac{\left(\rho-\rho_{1}\right)\left(2 \sigma_{1}-\sigma_{2}\right)}{2 r_{1}^{5}}+\frac{3 m_{2}}{m_{1}+m_{2}} \frac{\left(\rho-\rho_{2}\right)\left(2 \sigma_{1}{ }^{\prime}-\sigma_{2}{ }^{\prime}\right)}{2 r_{2}^{5}}+$

$\frac{3 m_{1}}{m_{1}+m_{2}} \frac{\left(\rho^{*}-\rho^{*}{ }_{1}\right)\left(\sigma_{1}-\sigma_{2}\right)}{r_{1}^{5}}+\frac{3 m_{2}}{m_{1}+m_{2}} \frac{\left(\rho^{*}-\rho_{2}^{*}\right)\left(\sigma_{1}{ }^{\prime}-\sigma_{2}{ }^{\prime}\right)}{r_{2}^{5}}-$

$\left.\left.\frac{15 m_{1}}{m_{1}+m_{2}} \frac{\left(\rho-\rho_{1}\right)\left(\sigma_{1}-\sigma_{2}\right) y^{2}}{2 r_{1}^{7}}-\frac{15 m_{2}}{m_{1}+m_{2}} \frac{\left(\rho-\rho_{2}\right)\left(\sigma_{1}{ }^{\prime}-\sigma_{2}{ }^{\prime}\right) y^{2}}{2 r_{2}^{7}}\right\}\right](2.27)$

Using

$r=\frac{a\left(1-e^{2}\right)}{1+e \cos v},=\frac{m_{2}}{m_{1}+m_{2}}, 1-\mu=\frac{m_{1}}{m_{1}+m_{2}}$,

The above expression will be reduced to the following form:

$\left(\frac{d^{2} \rho}{d v^{2}}\right)+2 i \frac{d \rho}{d v}=\frac{1}{1+e \cos v}\left[\rho-\frac{1}{n^{2}}\left\{\frac{(1-\mu)\left(\rho-\rho_{1}\right)}{r_{1}^{3}}+\frac{\mu\left(\rho-\rho_{2}\right)}{r_{2}^{3}}+\right.\right.$

$\frac{3(1-\mu)\left(\rho-\rho_{1}\right)\left(2 \sigma_{1}-\sigma_{2}\right)}{2 r_{1}^{5}}+\frac{3 \mu\left(\rho-\rho_{2}\right)\left(2 \sigma_{1}{ }^{\prime}-\sigma_{2}{ }^{\prime}\right)}{2 r_{2}^{5}}+\frac{3(1-\mu)\left(\rho^{*}-\rho^{*}{ }_{1}\right)\left(\sigma_{1}-\sigma_{2}\right)}{r_{1}^{5}}+$ $\frac{3 \mu\left(\rho^{*}-\rho^{*}{ }_{2}\right)\left(\sigma_{1}{ }^{\prime}-\sigma_{2}{ }^{\prime}\right)}{r_{2}^{5}}-\frac{15(1-\mu)\left(\rho-\rho_{1}\right)\left(\sigma_{1}-\sigma_{2}\right) y^{2}}{2 r_{1}^{7}}-$

$\left.\left.\frac{15 \mu\left(\rho-\rho_{2}\right)\left(\sigma_{1}{ }^{\prime}-\sigma_{2}{ }^{\prime}\right) y^{2}}{2 r_{2}^{7}}\right\}\right](2$

Replacing $=x+i y, \rho_{1}=x_{1}+i y_{1}, \rho_{2}=x_{2}+i y_{2}, \rho^{*}=i y, \rho_{1}^{*}=$ $i y_{1}, \rho_{2}^{*}=i y_{2}$ in the above Equation (2.28) and equating real and imaginary parts, we get:

$$
\begin{aligned}
\left(\frac{d^{2} x}{d v^{2}}\right)-2 \frac{d y}{d v}= & \frac{1}{1}+\operatorname{ecosv}[x \\
& -\frac{1}{n^{2}}\left\{\frac{(1-\mu)\left(x-x_{1}\right)}{r_{1}^{3}}+\frac{\mu\left(x-x_{2}\right)}{r_{2}{ }^{3}}\right. \\
& +\frac{3(1-\mu)\left(x-x_{1}\right)\left(2 \sigma_{1}-\sigma_{2}\right)}{2 r_{1}{ }^{\prime}} \\
& +\frac{3 \mu\left(x-x_{2}\right)\left(2 \sigma_{1}{ }^{\prime}-\sigma_{2}{ }^{\prime}\right)}{2 r_{2}^{5}} \\
& +-\frac{15(1-\mu)\left(x-x_{1}\right)\left(\sigma_{1}-\sigma_{2}\right) y^{2}}{2 r_{1}{ }^{2}} \\
& \left.\left.-\frac{\left.\left.15 \mu\left(x-x_{2}\right)\left(\sigma_{1}{ }^{\prime}-\sigma_{2}{ }^{\prime}\right) y^{2}\right)\right]}{2 r_{2}^{7}}\right\}\right]
\end{aligned}
$$

And

$\left(\frac{d^{2} y}{d v^{2}}\right)+2 \frac{d x}{d v}=\frac{1}{1+e \cos v}\left[y-\frac{1}{n^{2}}\left\{\frac{(1-\mu)\left(y-y_{1}\right)}{r_{1}^{3}}+\frac{\mu\left(y-y_{2}\right)}{r_{2}^{3}}+\right.\right.$ $\frac{3(1-\mu)\left(y-y_{1}\right)\left(2 \sigma_{1}-\sigma_{2}\right)}{2 r_{1}^{5}}+\frac{3 \mu\left(y-y_{2}\right)\left(2 \sigma_{1}{ }^{\prime}-\sigma_{2}{ }^{\prime}\right)}{2 r_{2}^{5}}+\frac{3(1-\mu)\left(y-y_{1}\right)\left(\sigma_{1}-\sigma_{2}\right)}{r_{1}^{5}}+$ $\left.\left.\frac{3 \mu\left(y-y_{2}\right)\left(\sigma_{1}{ }^{\prime}-\sigma_{2}{ }^{\prime}\right)}{r_{2}^{5}}-\frac{15(1-\mu)\left(y-y_{1}\right)\left(\sigma_{1}-\sigma_{2}\right) y^{2}}{2 r_{1}^{7}}-\frac{15 \mu\left(y-y_{2}\right)\left(\sigma_{1}{ }^{\prime}-\sigma_{2}{ }^{\prime}\right) y^{2}}{2 r_{2}^{7}}\right\}\right]$ 
Hence the equation of motion of infinitesimal shall be reduced to the following form by replacing

$$
\begin{aligned}
x_{1}=-\mu, x_{2}=1-\mu & y_{1}=0, y_{2}=0 \\
\left(\frac{d^{2} x}{d v^{2}}\right)-2 \frac{d y}{d v}= & \frac{1}{1+e \cos v}[x \\
& -\frac{1}{n^{2}}\left\{\frac{(1-\mu)(x+\mu)}{r_{1}^{3}}+\frac{\mu(x-1+\mu)}{r_{2}^{3}}\right. \\
& +\frac{3(1-\mu)(x+\mu)\left(2 \sigma_{1}-\sigma_{2}\right)}{2 r_{1}^{5}} \\
& +\frac{3 \mu(x-1+\mu)\left(2 \sigma_{1}{ }^{\prime}-\sigma_{2}{ }^{\prime}\right)}{2 r_{2}^{5}} \\
& -\frac{15(1-\mu)(x+\mu)\left(\sigma_{1}-\sigma_{2}\right) y^{2}}{2 r_{1}^{7}} \\
& \left.\left.-\frac{\left.15 \mu(x-1+\mu)\left(\sigma_{1}{ }^{\prime}-\sigma_{2}{ }^{\prime}\right) y^{2}\right)}{2 r_{2}^{7}}\right\}\right]
\end{aligned}
$$

And

$\left(\frac{d^{2} y}{d v^{2}}\right)+2 \frac{d x}{d v}=\frac{1}{1+e \cos v}\left[y-\frac{1}{n^{2}}\left\{\frac{(1-\mu) y}{r_{1}^{3}}+\frac{\mu y}{r_{2}^{3}}+\frac{3(1-\mu) y\left(2 \sigma_{1}-\sigma_{2}\right)}{2 r_{1}^{5}}+\right.\right.$ $\frac{3 \mu y\left(2 \sigma_{1}{ }^{\prime}-\sigma_{2}{ }^{\prime}\right)}{2 r_{2}^{5}}+\frac{3(1-\mu) y\left(\sigma_{1}-\sigma_{2}\right)}{r_{1}^{5}}+\frac{3 \mu y\left(\sigma_{1}{ }^{\prime}-\sigma_{2}{ }^{\prime}\right)}{r_{2}^{5}}-$

$\left.\left.\frac{15(1-\mu)\left(\sigma_{1}-\sigma_{2}\right) y^{3}}{2 r_{1}^{7}}-\frac{15 \mu\left(\sigma_{1}{ }^{\prime}-\sigma_{2}{ }^{\prime}\right) y^{3}}{2 r_{2}^{7}}\right\}\right](2.30)$

The differential equation of motion of the third body $P$ in nondimensional barycentre, pulsating and non-uniformly rotating coordinate system $(x, y)$ is written in the form:

$$
\begin{aligned}
x^{\prime \prime}-2 y^{\prime \prime} & =\frac{1}{1+e \cos v}\left(\frac{\partial \Omega}{\partial x}\right) \\
y^{\prime \prime}+2 x^{\prime \prime} & =\frac{1}{1+e \cos v}\left(\frac{\partial \Omega}{\partial y}\right)
\end{aligned}
$$

Where ' denotes differentiation with respect to $v$, and

$$
\begin{aligned}
& \Omega=\frac{x^{2}+y^{2}}{2}+\frac{1}{n^{2}}\left[\frac{(1-\mu)}{r_{1}}+\frac{\mu}{r_{2}}+\frac{(1-\mu)\left(2 \sigma_{1}-\sigma_{2}\right)}{2 r_{1}^{3}}+\frac{\mu\left(2 \sigma_{1}{ }^{\prime}-\sigma_{2}{ }^{\prime}\right)}{2 r_{2}^{3}}-\right. \\
& \left.\frac{3(1-\mu)\left(\sigma_{1}-\sigma_{2}\right) y^{2}}{2 r_{1}^{5}}-\frac{3 \mu\left(\sigma_{1}{ }^{\prime}-\sigma_{2}{ }^{\prime}\right) y^{2}}{2 r_{2}^{5}}\right]
\end{aligned}
$$

Where

$$
n^{2}=1+\frac{3}{2}\left(2 \sigma_{1}-\sigma_{2}\right)+\frac{3}{2}\left(2 \sigma_{1}^{\prime}-\sigma_{2}^{\prime}\right)
$$

And

$$
\begin{gathered}
r_{1}^{2}=(x+\mu)^{2}+y^{2} \\
r_{2}^{2}=(x-1+\mu)^{2}+y^{2}
\end{gathered}
$$

Thus we have the equation of motion of an elliptic restricted three body problem in which both the primaries are oblate and triaxial.

\section{Location of equilibrium points}

The equilibrium points of the system are the stationary points, so are given by the equation

$$
\frac{\partial \Omega}{\partial x}=\frac{\partial \Omega}{\partial y}=0
$$

where $\Omega$ is given in equation (2.32), so we get:

$$
\begin{aligned}
& \frac{\partial \Omega}{\partial x}=x-\frac{1}{n^{2}}\left[\frac{(1-\mu)(x+\mu)}{r_{1}^{3}}+\frac{\mu(x-1+\mu)}{r_{2}^{3}}+\frac{3(1-\mu)(x+\mu)\left(2 \sigma_{1}-\sigma_{2}\right)}{2 r_{1}^{5}}+\right. \\
& \frac{3 \mu(x-1+\mu)\left(2 \sigma_{1}{ }^{\prime}-\sigma_{2}{ }^{\prime}\right)}{2 r_{2}^{5}}-\frac{15(1-\mu)(x+\mu)\left(\sigma_{1}-\sigma_{2}\right) y^{2}}{2 r_{1}^{7}}- \\
& \left.\frac{15 \mu(x-1+\mu)\left(\sigma_{1}{ }^{\prime}-\sigma_{2}{ }^{\prime}\right) y^{2}}{2 r_{2}^{7}}\right]=0(3.2)
\end{aligned}
$$

And

$\frac{\partial \Omega}{\partial y}=y\left[1-\frac{1}{n^{2}}\left\{\frac{(1-\mu)}{r_{1}^{3}}+\frac{\mu}{r_{2}^{3}}+\frac{3(1-\mu)\left(4 \sigma_{1}-3 \sigma_{2}\right)}{2 r_{1}^{5}}+\frac{3 \mu\left(4 \sigma_{1}{ }^{\prime}-3 \sigma_{2}{ }^{\prime}\right)}{2 r_{2}^{5}}-\right.\right.$

$\left.\left.\frac{15(1-\mu)\left(\sigma_{1}-\sigma_{2}\right) y^{2} q_{1}}{2 r_{1}^{7}}-\frac{15 \mu\left(\sigma_{1}{ }^{\prime}-\sigma_{2}{ }^{\prime}\right) y^{2} q_{2}}{2 r_{2}^{7}}\right\}\right](3.3)$

Since $y \neq 0$,we have from equation(3.3):

$1-\frac{1}{n^{2}}\left[\frac{(1-\mu)}{r_{1}^{3}}+\frac{\mu}{r_{2}^{3}}+\frac{3(1-\mu)\left(4 \sigma_{1}-3 \sigma_{2}\right)}{2 r_{1}^{5}}+\frac{3 \mu\left(4 \sigma_{1}{ }^{\prime}-3 \sigma_{2}{ }^{\prime}\right)}{2 r_{2}^{5}}-\right.$

$\left.\frac{15(1-\mu)\left(\sigma_{1}-\sigma_{2}\right) y^{2}}{2 r_{1}^{7}}-\frac{15 \mu\left(\sigma_{1}{ }^{\prime}-\sigma_{2}{ }^{\prime}\right) y^{2}}{2 r_{2}^{7}}\right]=0$

Using the above equation (3.2) and (3.4), we get:

$\left[-n^{2}(1-\mu)+\frac{(1-\mu)}{r_{1}^{3}}-\frac{15(1-\mu)\left(\sigma_{1}-\sigma_{2}\right) y^{2}}{2 r_{1}^{7}}-\frac{15 \mu\left(\sigma_{1}{ }^{\prime}-\sigma_{2}{ }^{\prime}\right) y^{2}}{2 r_{2}^{7}}+\right.$

$\frac{3(1-\mu)\left(2 \sigma_{1}-\sigma_{2}\right)}{2 r_{1}^{5}}-\frac{3(1-\mu)(x-1+\mu)\left(\sigma_{1}-\sigma_{2}\right)}{2 r_{1}^{5}}+\frac{3 \mu\left(2 \sigma_{1}{ }^{\prime}-\sigma_{2}{ }^{\prime}\right)}{2 r_{2}^{5}}-$

$\left.\frac{3 \mu\left(\sigma_{1}{ }^{\prime}-\sigma_{2}{ }^{\prime}\right)(x-1+\mu)}{r_{2}^{5}}\right]=0(3.5)$

And

$-\mu+\frac{1}{n^{2}}\left[\frac{\mu}{r_{2}^{3}}+\frac{3(1-\mu)(x+\mu)\left(\sigma_{1}-\sigma_{2}\right)}{r_{1}^{5}}+\frac{3 \mu(x+\mu)\left(\sigma_{1}{ }^{\prime}-\sigma_{2}{ }^{\prime}\right)}{r_{2}^{5}}+\right.$ $\left.\frac{3 \mu\left(2 \sigma_{1}{ }^{\prime}-\sigma_{2}{ }^{\prime}\right)}{2 r_{2}^{5}}-\frac{15 \mu\left(\sigma_{1}{ }^{\prime}-\sigma_{2}{ }^{\prime}\right) y^{2}}{2 r_{2}^{7}}\right]=0(3.6)$

$\sigma_{1}, \sigma_{2}, \sigma_{1}{ }^{\prime}$ and $\sigma_{2}{ }^{\prime}$ are small quantities hence we assume that the solution of the above equation if $\sigma_{1} \neq 0, \sigma_{2} \neq 0, \sigma_{1}{ }^{\prime} \neq 0$ and $\sigma_{2}{ }^{\prime} \neq 0$ as

$r_{1}=1+\epsilon_{1} r_{2}=1+\epsilon_{2}$

where $\epsilon_{1}$ and $\epsilon_{2}$ are less than 1 .

Substituting the values of $r_{1}$ and $r_{2}$ in equation (2.34) we get:

$$
\begin{gathered}
x=\frac{1}{2}-\mu+\left(\epsilon_{1}+\epsilon_{2}\right) \\
y= \pm \frac{\sqrt{3}}{2}\left[1+\frac{2}{3}\left(\epsilon_{1}+\epsilon_{2}\right)\right]
\end{gathered}
$$

Further simplifying the coordinates of triangular equilibrium points are given by:

$$
\begin{aligned}
& x=\frac{1}{2}-\mu++\left[\frac{-3}{8}-\frac{(1-\mu)}{2 \mu}\right] \sigma_{1}+\left[\frac{7}{8}+\frac{(1-\mu)}{2 \mu}\right] \sigma_{2} \\
& +\left[\frac{3}{8}-\frac{3 \mu}{8(1-\mu)}\right] \sigma_{1}{ }^{\prime}+\left[\frac{-7}{8}+\frac{7 \mu}{8(1-\mu)}\right] \sigma_{2}{ }^{\prime} \\
& y= \pm\left[1+\frac{2}{3}\left\{\left[\frac{-19}{8} \mp \frac{(1-\mu)}{2 \mu}-\right] \sigma_{1}\left[\frac{15}{8}-\frac{(1-\mu)}{2 \mu}\right] \sigma_{2}+\left[\frac{-19}{8}-\right.\right.\right. \\
& \left.\left.\left.\frac{3 \mu}{8(1-\mu)}\right] \sigma_{1}{ }^{\prime}+\left[\frac{15}{8}+\frac{7 \mu}{8(1-\mu)}\right] \sigma_{2}{ }^{\prime}\right\}\right](3.9)
\end{aligned}
$$

\section{Different curves of zero velocity}

In order to derive curves of zero velocity of the infinitesimal mass in elliptical restricted three body problem considering the triaxiality of both the primaries, multiply equation (2.31) by $\mathrm{x}$ ' and equation (2.32) byy 'and further add both the equation. Then we obtain

$$
x^{\prime} x^{\prime \prime}+y^{\prime} y^{\prime \prime}=\frac{\partial \Omega}{\partial x} x^{\prime}+\frac{\partial \Omega}{\partial x} y^{\prime}
$$




$$
\frac{1}{2} \frac{\partial}{\partial v}\left[x^{\prime 2}+y^{\prime 2}\right]=\frac{\partial \Omega}{\partial v}
$$

Equation (4.2) can be integrated to give

$$
\frac{1}{2}\left[x^{\prime 2}+y^{\prime 2}\right]=\int \frac{\partial \Omega}{1+e \cos v}+c
$$

The presence of the term $(1+\mathrm{ecosv})$ in the denominator of equation (4.3) gives problem in integrating equation (4.3) to any defined form. The Jacobi integral of the classical circular problem is not adjusted in elliptical restricted three body problem in usual sense. In elliptical restricted three body problem Jacobi integral does not exist and energy along any of the orbit depends on time. Now, consider the potential function which is represented as follows:

$$
\varphi(x, y)=\frac{\Omega(x, y)}{1+e \cos v}+c
$$

So, $\Omega(x, y)$ depends on the position coordinate of the as well as the independent variable of the infinitesimal mass. In elliptical The Jacobi constant is defined as

$$
\begin{aligned}
& x^{\prime 2}+y^{\prime 2}-\frac{1}{1+e \cos v}\left[\frac{x^{2}+y^{2}}{2}+\frac{1}{n^{2}}\left[\frac{(1-\mu)}{r_{1}}+\frac{\mu}{r_{2}}+\frac{(1-\mu)\left(2 \sigma_{1}-\sigma_{2}\right)}{2 r_{1}^{3}}+\right.\right. \\
& \left.\left.\frac{\mu\left(2 \sigma_{1}{ }^{\prime}-\sigma_{2}{ }^{\prime}\right)}{2 r_{2}^{3}}-\frac{3(1-\mu)\left(\sigma_{1}-\sigma_{2}\right) y^{2}}{2 r_{1}^{5}}-\frac{3 \mu\left(\sigma_{1}{ }^{\prime}-\sigma_{2}{ }^{\prime}\right) y^{2}}{2 r_{2}^{5}}\right]\right]=C(4.5)
\end{aligned}
$$

The above equation (4.5) describes the curve of zero velocity. The zero velocity curves pulsate with the frequency of the elliptical motion of the infinitesimal mass in elliptical restricted three body problem. Hence, in planar elliptical restricted three body problem, the zero velocity curves is given as:

$\left[\frac{x^{2}+y^{2}}{2}+\frac{1}{n^{2}}\left[\frac{(1-\mu)}{r_{1}}+\frac{\mu}{r_{2}}+\frac{(1-\mu)\left(2 \sigma_{1}-\sigma_{2}\right)}{2 r_{1}^{3}}+\frac{\mu\left(2 \sigma_{1}{ }^{\prime}-\sigma_{2}{ }^{\prime}\right)}{2 r_{2}^{3}}-\right.\right.$

$\left.\left.\frac{3(1-\mu)\left(\sigma_{1}-\sigma_{2}\right) y^{2}}{2 r_{1}^{5}}-\frac{3 \mu\left(\sigma_{1}{ }^{\prime}-\sigma_{2}{ }^{\prime}\right) y^{2}}{2 r_{2}^{5}}\right]\right]=C^{*}(4.6)$

where

$$
C^{*}=C(1+e \cos v)
$$

The zero velocity curve is plotted in Figs.1-26 by varying the parameters.

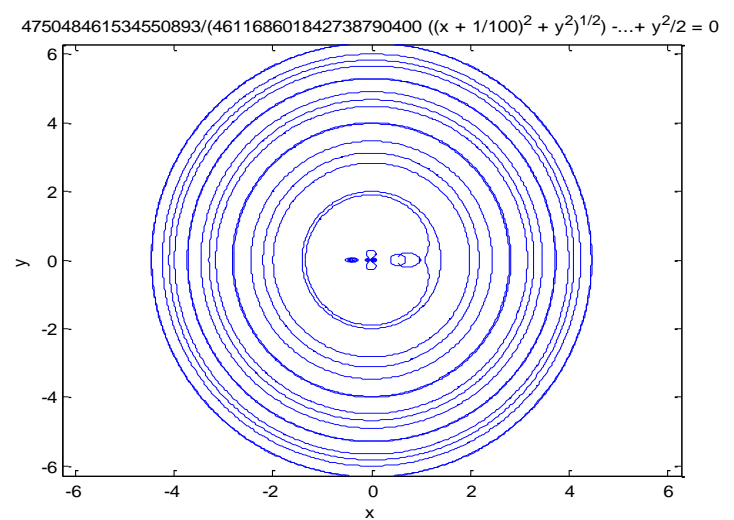

Fig. 1:Zero Velocity Curve when $\mu=.01$, $\sigma_{1}=0.003 ; \sigma_{2}=.0002 ; \sigma^{\prime}{ }_{1}=0.0007 ; \sigma^{\prime}{ }_{2}=0.0004$.

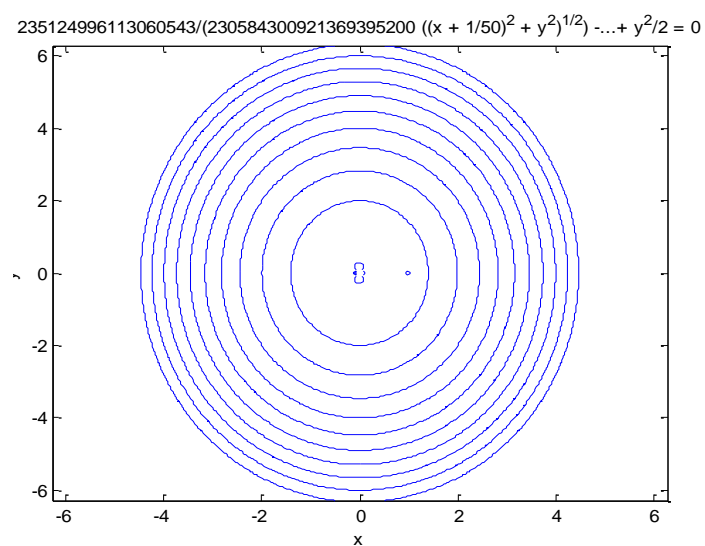

Fig. 2:Zero Velocity Curve when $\mu=.02$, $\sigma_{1}=0.003 ; \sigma_{2}=.0002 ; \sigma^{\prime}{ }_{1}=0.0007 ; \sigma^{\prime}{ }_{2}=0.0004$

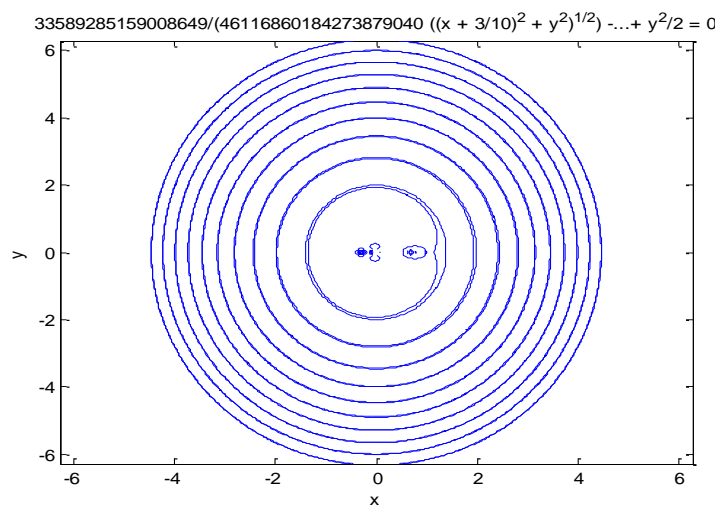

Fig. 3:Zero Velocity Curve When $\mu=0.3$, $\sigma_{1}=0.003 ; \sigma_{2}=.0002 ; \sigma_{1}^{\prime}=0.0007 ; \sigma^{\prime}{ }_{2}=0.0004$.

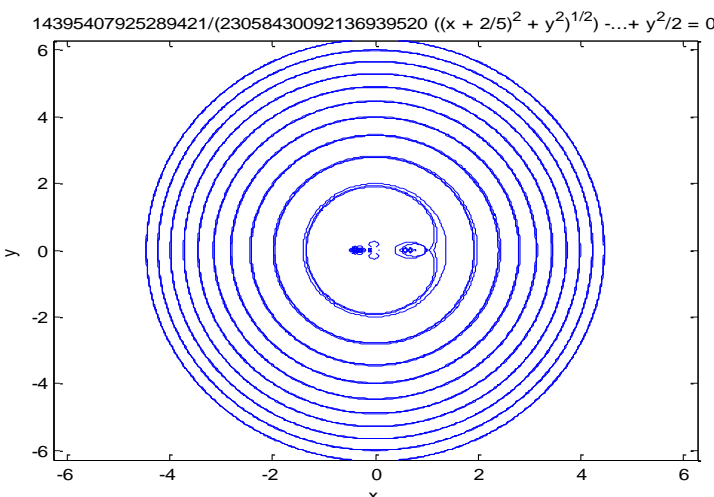

Fig. 4:Zero Velocity Curve When $\mu=0.4, \sigma_{1}=0.003 ; \sigma_{2}=.0002$; $\sigma_{1}^{\prime}=0.0007 ; \sigma_{2}^{\prime}=0.0004$

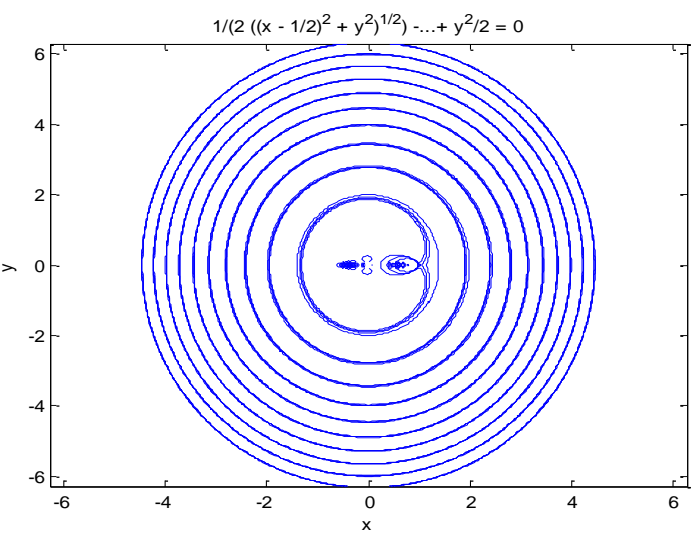

Fig. 5:Zero Velocity Curve when $\mu=0.5, \sigma_{1}=0.003 ; \sigma_{2}=.0002 ; \sigma_{1}^{\prime}=0.0007$; $\sigma_{2}^{\prime}=0.0004$ 


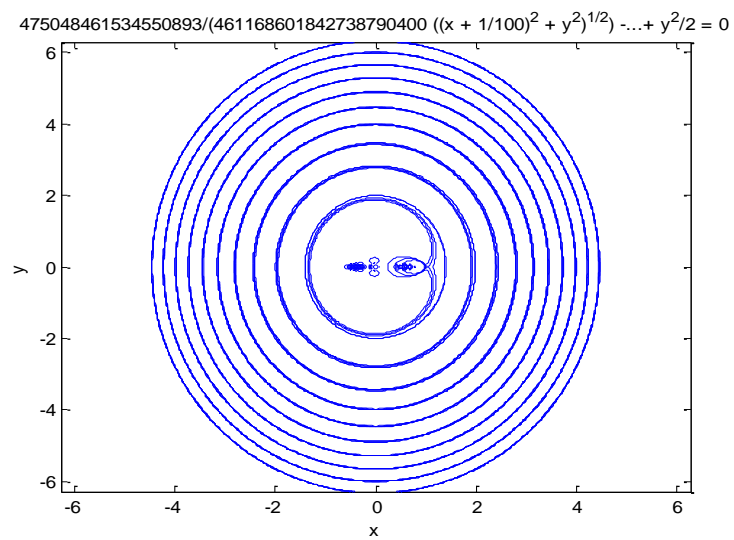

Fig .6:Zero Velocity Curve when $\mu=0.01, \sigma_{1}=0.0005 ; \sigma_{2}=.0002$; $\sigma_{1}^{\prime}=0.0007 ; \sigma_{2}^{\prime}=0.0004$.

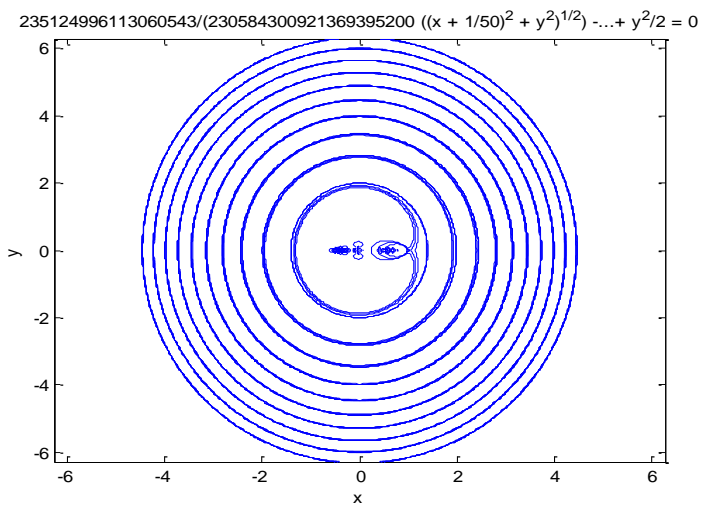

Fig. 7:Zero Velocity Curve when $\mu=0.02, \sigma_{1}=0.0005 ; \sigma_{2}=.0002$; $\sigma^{\prime}{ }_{1}=0.0007 ; \sigma_{2}^{\prime}=0.0004$.

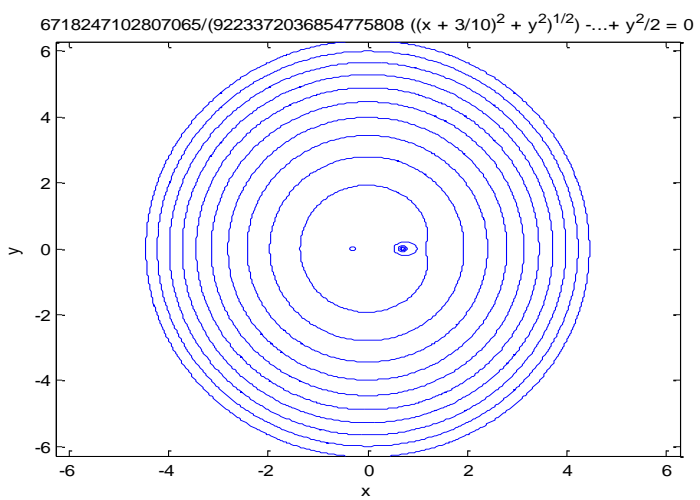

Fig. 8:Zero Velocity Curve when $\mu=0.3$ $\sigma_{1}=0.0005 ; \sigma_{2}=.0002 ; \sigma_{1}^{\prime}=0.0003 ; \sigma_{2}^{\prime}=0.0002$.

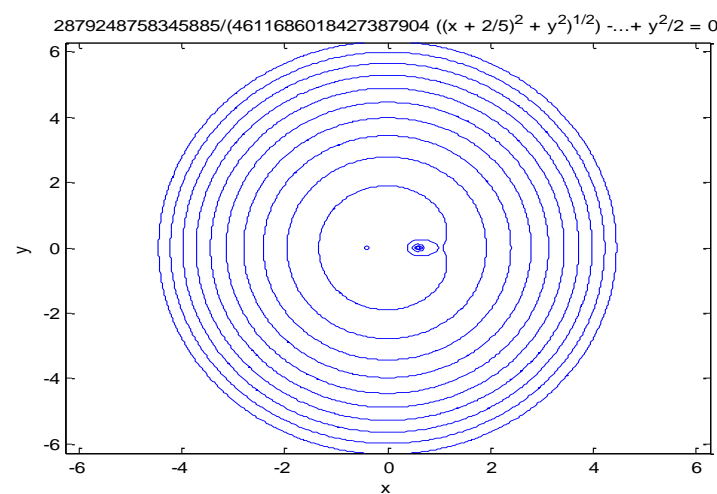

Fig. 9:Zero Velocity Curve When $\mu=0.4$, $\sigma_{1}=0.0005 ; \sigma_{2}=.0002 ; \sigma_{1}^{\prime}=0.0003 ; \sigma_{2}^{\prime}=0.0002$.

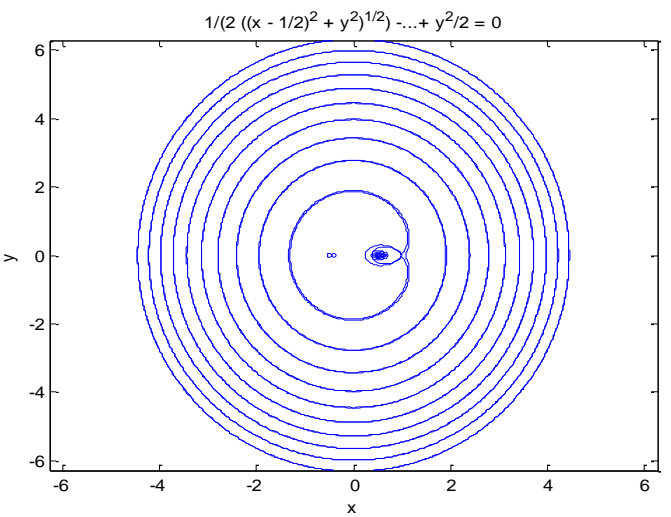

Fig. 10:Zero Velocity Curve when $\mu=0.5$, $\sigma_{1}=0.0005 ; \sigma_{2}=.0002 ; \sigma^{\prime}{ }_{1}=0.0003 ; \sigma^{\prime}{ }_{2}=0.0002$.

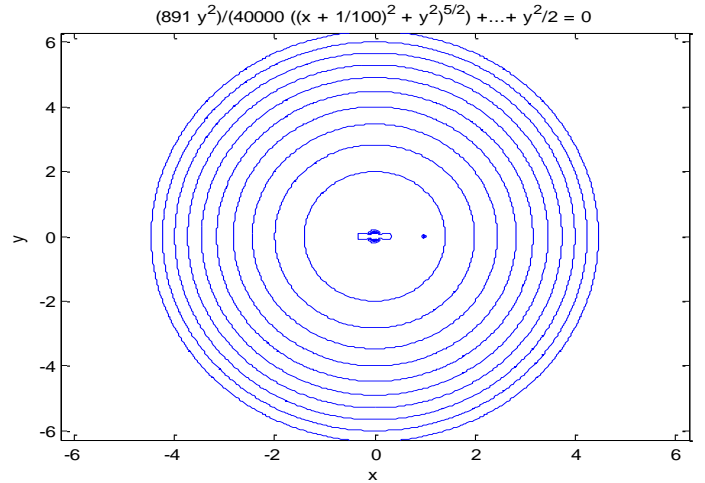

Fig. 11:Zero Velocity Curve when $\mu=0.01$, $\sigma_{1}=0.005 ; \sigma_{2}=.002 ; \sigma_{1}^{\prime}=0.0005 ; \sigma_{2}^{\prime}=0.02$.

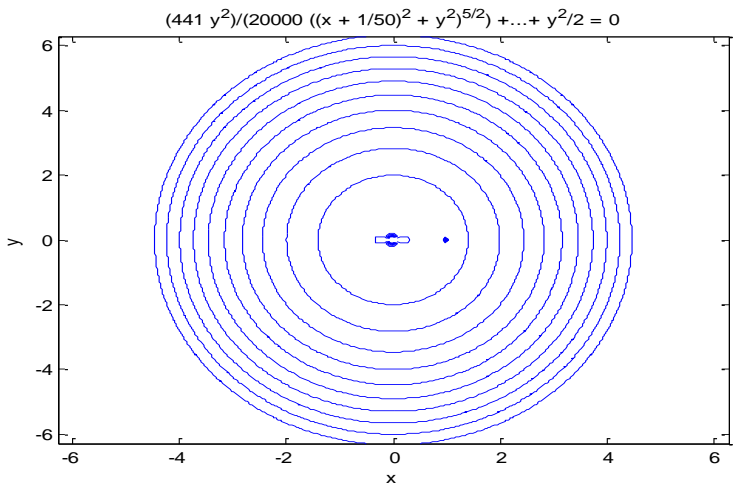

Fig. 12:Zero Velocity Curve when $\mu=0.02$, $\sigma_{1}=0.005 ; \sigma_{2}=.02 ; \sigma_{1}^{\prime}=0.0005 ; \sigma_{2}^{\prime}=0.02$.

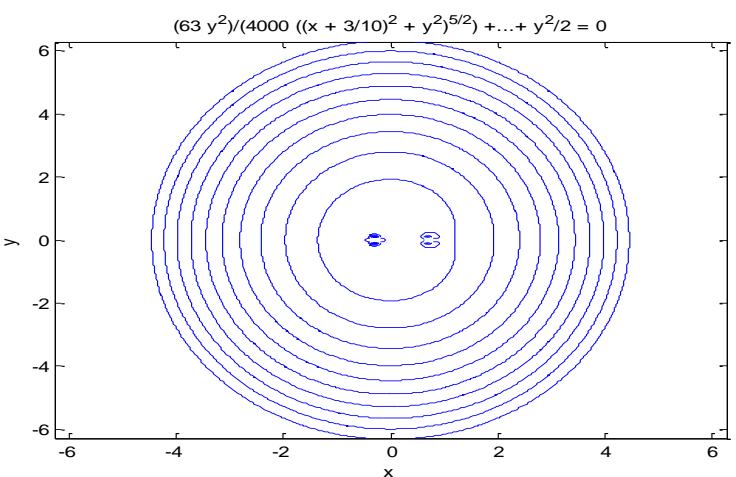

Fig. 13:Zero Velocity Curve when $\mu=0.3$, $\sigma_{1}=0.005 ; \sigma_{2}=.02 ; \sigma_{1}^{\prime}=0.0005 ; \sigma_{2}^{\prime}=0.02$. 


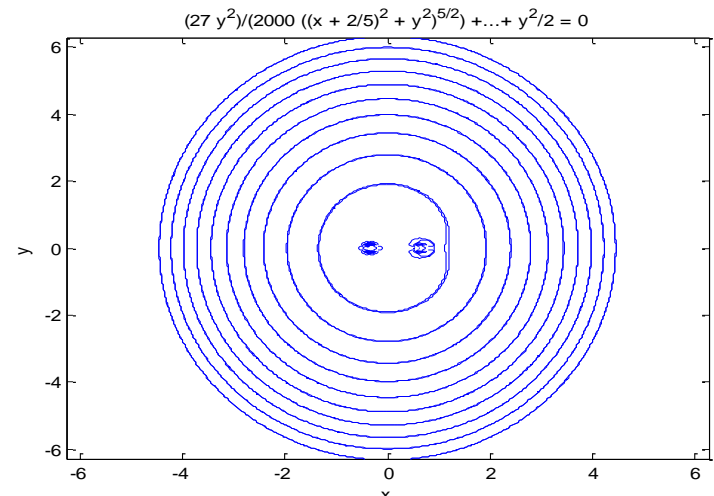

Fig. 14:Zero Velocity Curve when $\mu=0.4, \sigma_{1}=0.005 ; \sigma_{2}=.02 ; \sigma^{\prime}{ }_{1}=0.0005$; $\sigma_{2}^{\prime}=0.02$.

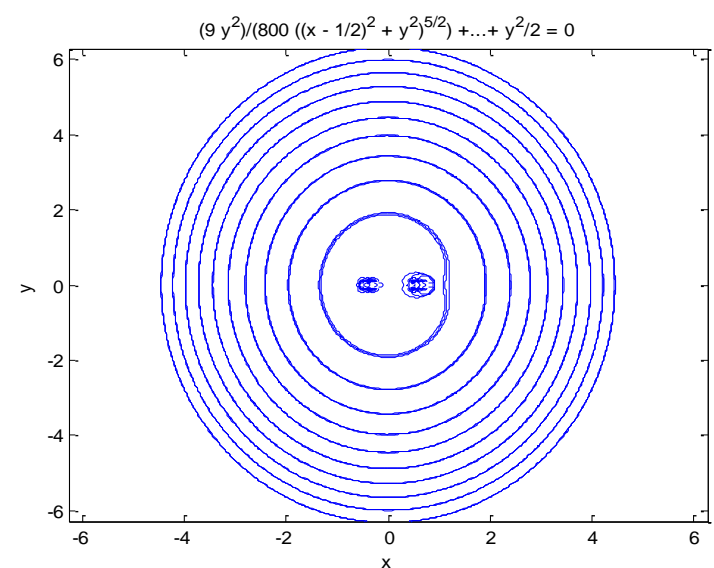

Fig. 15:Zero Velocity Curve when $\mu=0.5, \sigma_{1}=0.005 ; \sigma_{2}=.02 ; \sigma_{1}^{\prime}=0.0005$; $\sigma_{2}^{\prime}=0.02$.

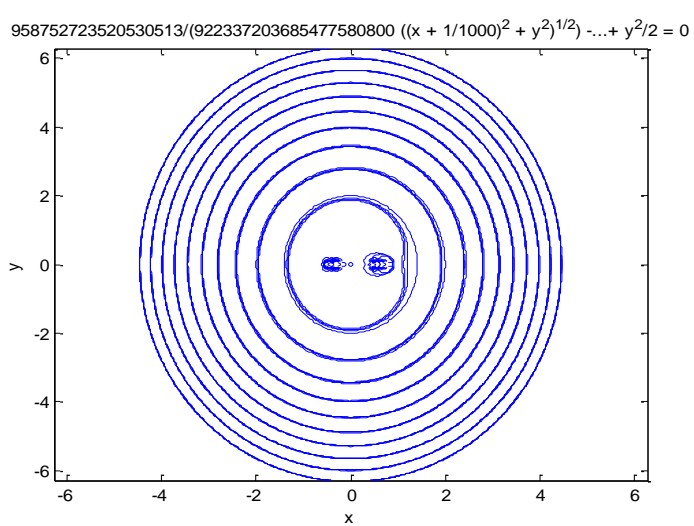

Fig. 16:Zero Velocity Curve when $\mu=0.001$, $\sigma_{1}=0.0003 ; \sigma_{2}=.0002 ; \sigma_{1}^{\prime}=0.0005 ; \sigma^{\prime}{ }_{2}=0.0002$.

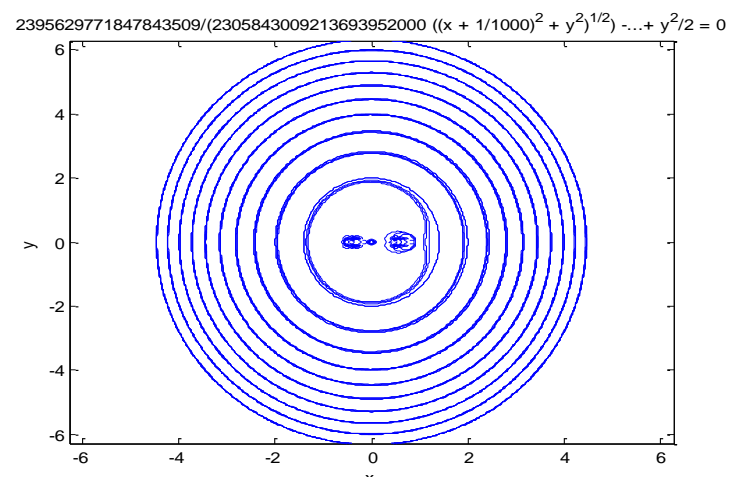

Fig. 17:Zero Velocity Curve when $\mu=0.001, \sigma_{1}=0.003 ; \sigma_{2}=.002$; $\sigma_{1}^{\prime}=0.005 ; \sigma_{2}^{\prime}=0.002$.

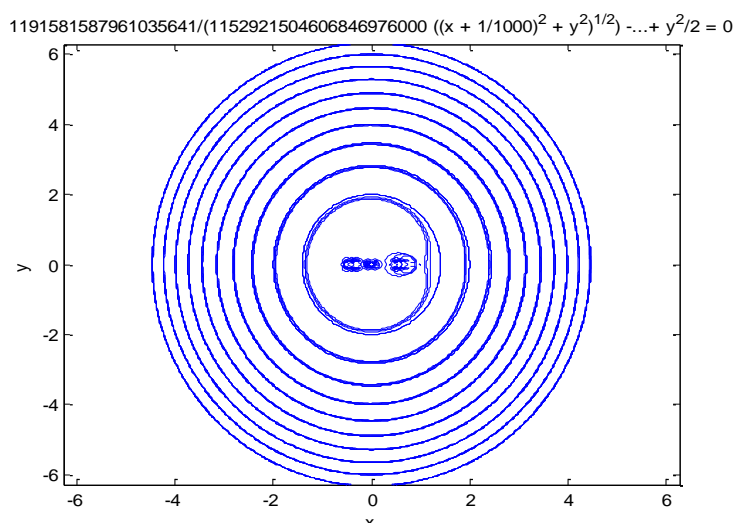

Fig. 18:Zero Velocity Curve when $\mu=0.001, \sigma_{1}=0.03 ; \sigma_{2}=.02 ; \sigma_{1}^{\prime}=0.05$; $\sigma_{2}^{\prime}=0.02$.

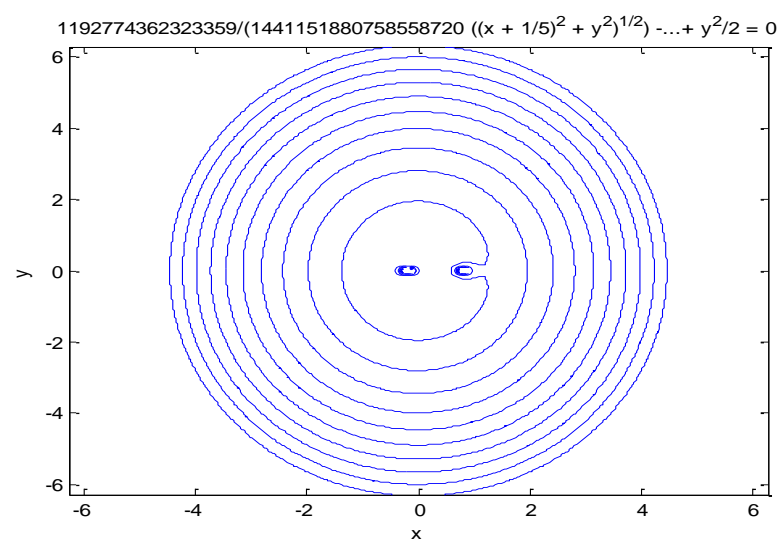

Fig. 19:Zero Velocity Curve when $\mu=0.2, \sigma_{1}=0.03 ; \sigma_{2}=.02 ; \sigma_{1}^{\prime}=0.05$; $\sigma_{2}^{\prime}=0.02$.

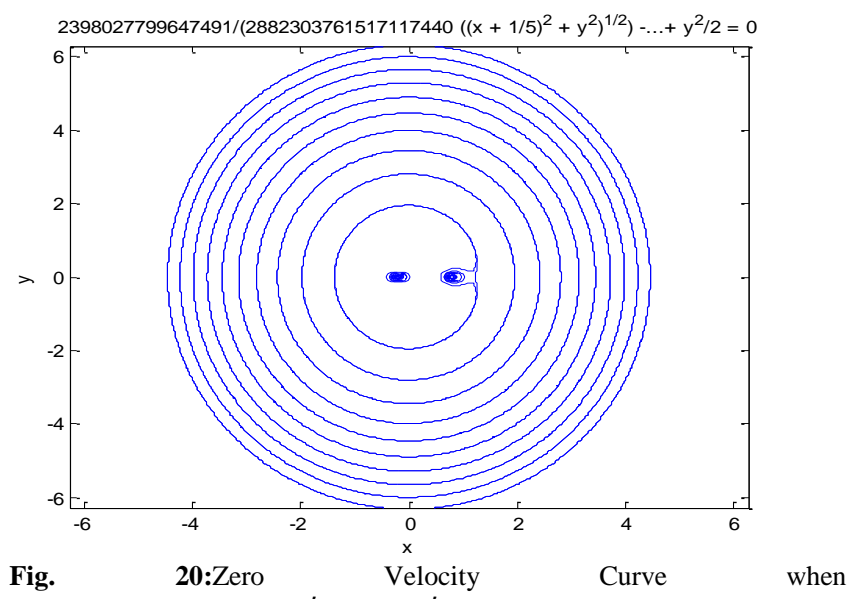
$\mu=0.2, \sigma_{1}=0.003 ; \sigma_{2}=.002 ; \sigma^{\prime}{ }_{1}=0.005 ; \sigma^{\prime}{ }_{2}=0.002$.

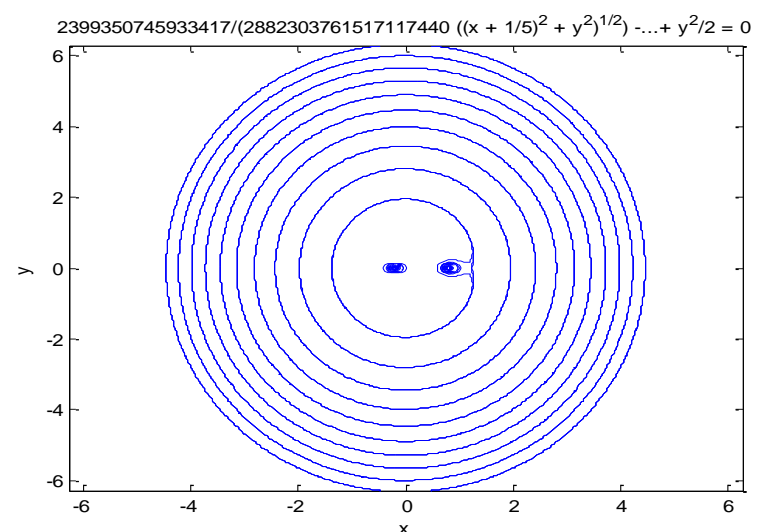

Fig. 21:Zero Velocity Curve when $\mu=0.2, \sigma_{1}=0.0002 ; \sigma_{2}=.0001$; $\sigma^{\prime}{ }_{1}=0.0003 ; \sigma_{2}^{\prime}=0.0002$. 


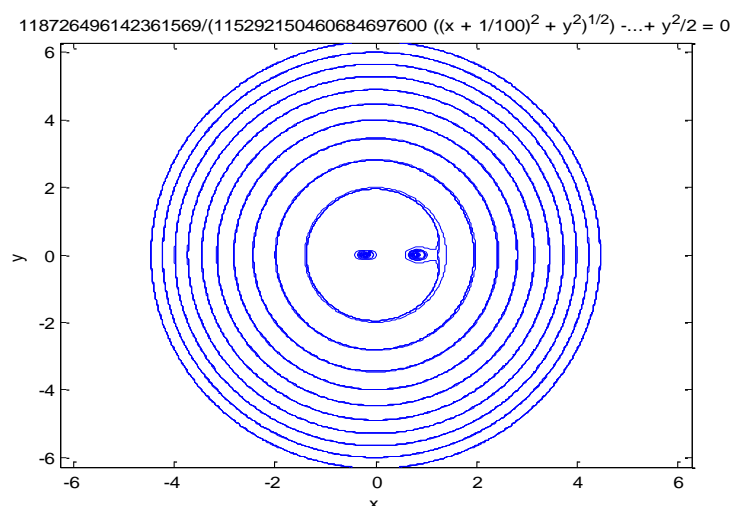

Fig. 22:Zero Velocity Curve when $\mu=0.01, \sigma_{1}=0.0002 ; \sigma_{2}=.0001$; $\sigma_{1}^{\prime}=0.0003 ; \sigma_{2}^{\prime}=0.0002$.

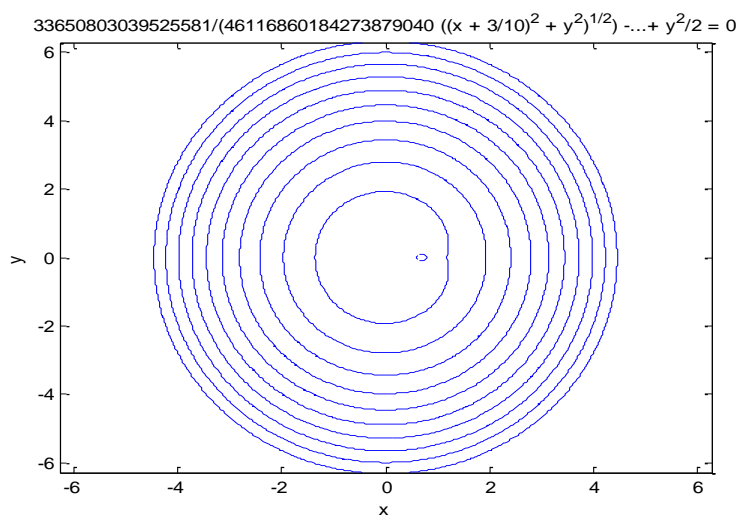

Fig. 23:Zero Velocity Curve when $\mu=0.3, \sigma_{1}=0.0002 ; \sigma_{2}=.0001$; $\sigma_{1}^{\prime}=0.0003 ; \sigma_{2}^{\prime}=0.002$.

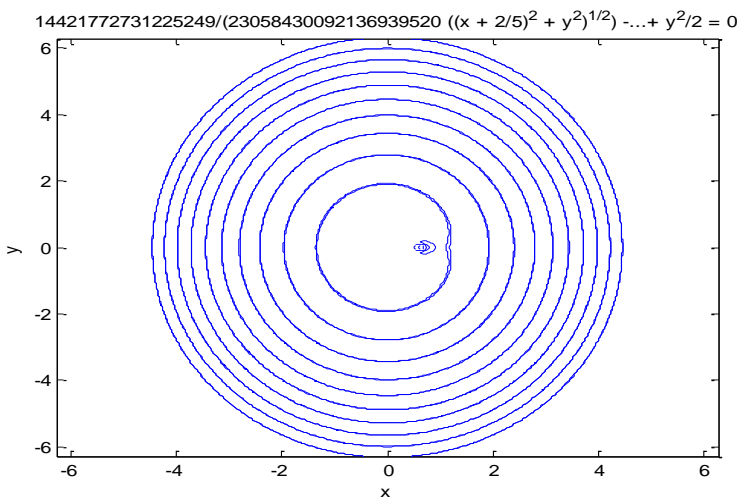

Fig. 24:Zero Velocity Curve when $\mu=0.4, \sigma_{1}=0.0002 ; \sigma_{2}=.0001$; $\sigma^{\prime}{ }_{1}=0.003 ; \sigma_{2}^{\prime}=0.002$.

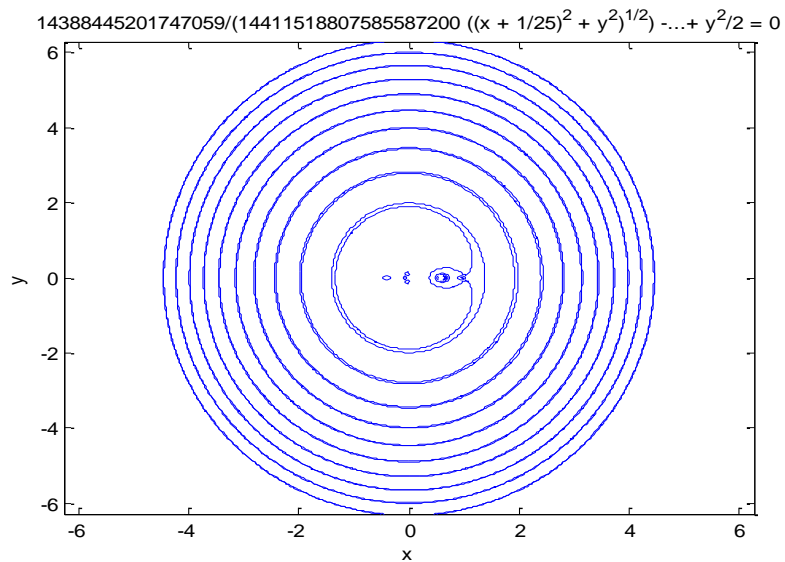

Fig. 25:Zero Velocity Curve when $\mu=0.04, \sigma_{1}=0.0008 ; \sigma_{2}=.0002$ $\sigma_{1}^{\prime}=0.004 ; \sigma_{2}^{\prime}=0.002$.

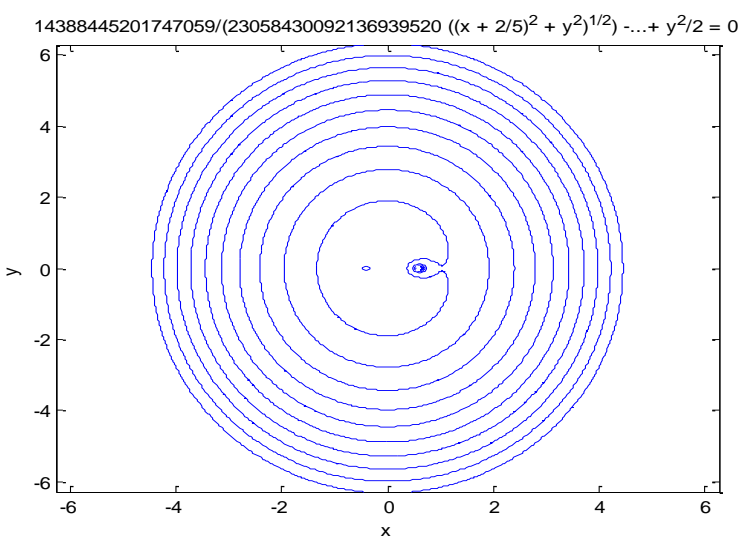

Fig. 26:Zero Velocity Curve when $\mu=0.4, \sigma_{1}=0.0008 ; \sigma_{2}=.0002$; $\sigma_{1}^{\prime}=0.004 ; \sigma_{2}^{\prime}=0.002$.

\section{Discussion and results}

The differential equation governing the motion of the triangular points in elliptical restricted three body has been derived considering the oblate and triaxial rigid body. The problem is model under the assumption that the eccentricity of the orbit of the gravitating bodies is small.

The zero velocity curves considering oblateness and triaxiliaty of the rigid body has been studied using simulation technique by varying the parameters. From Fig 1-26 it has been observed that oblateness and triaxiliaty of either or both the primaries affect the nature of zero velocity curves. From Fig 1-5 it is clear that by changing the mass ratio and by considering the other parameters as constant there is change in the nature of zero velocity curve.

\section{References}

[1] Danby JMA (1964), Stability of the triangular points in the elliptical restricted problem of three bodies, Astronomical Journal, 69,166-174.

[2] Bennet A (1965), Characteristic exponents of the five equilibrium solutions in the EllipticallyRestricted Problem, ICARUS 4, $177-$ 187.https://doi.org/10.1016/0019-1035(65)90060-6.

[3] Szebehely V (1975), Stability of the points of equilibrium in restricted problem, Astronomical Journal, 72, 7 9.https://doi.org/10.1086/110195.

[4] Szebehely V (1967), Theory of Orbits, Academic press, New-York.

[5] Markeev A P (1978), Libration points in celestial mechanics and cosmic dynamics, NAUKA Moscow, 312.

[6] Grebnikov E A (1964), On the stability of the Lagrangian triangle solutions of the restricted elliptic three body problem, Soviet Astronomy, 8 , No.3, 567-578.

[7] Grebnikov E A (1986), the methods of Averaging Applications, NAUKA, Moscow Revised.

[8] Gyorgyey J (1985), on the non-linear motions around the elliptical restricted problem of three bodies, Celestial Mechanics and Dynamical Astronomy, 36, No.3, 281285.https://doi.org/10.1007/BF01230741.

[9] KumarV \&ChoudharyRK (1990), Non-linear stability of the triangular libration points for the photogravitationalelliptic restrictedproblem of three bodies, Celestial Mechanics and Dynamical Astronomy, 48, No.4, 299-317.https://doi.org/10.1007/BF00049387.

[10] Kumar S \&Ishwar B (2009), Solutions of Generalized Photogravitational Elliptical Restricted ThreeBody Problem, AIP Conf.Proc, 1146,456.https://doi.org/10.1063/1.3183564.

[11] MarkellosV V, PerdiosE \&LabrapoulouP(1992), Linear stability of the triangular equilibrium points in the photogravitationalelliptic restrictedthree body problem, Astrophysics and Space Science ,194, 207-214.https://doi.org/10.1007/BF00643991.

[12] ZimvoschikovA S \&Thkai V N (2004), Instability of libration points and resonance phenomena in the photogravitationalellipticalrestricted three bodyproblem, Solar system research, 38(2), 155163.https://doi.org/10.1023/B:SOLS.0000022826.31475.a7.

[13] AmmarMK (2008), The effect of solar radiation pressure on the Lagrangian points in the elliptical restricted three body problem, 
Astrophysics and Space Science, 313 , 393408.https://doi.org/10.1007/s10509-007-9709-z.

[14] Singh, J \&Aishetu U (2012a), Motion in the photogravitationalellipticrestrictedthree body problem under an oblate primary, Astronomical Journal, 143, No.5, 109.https://doi.org/10.1088/00046256/143/5/109.

[15] Singh J \&Aishetu U (2012b), On the stability of triangular points in the ellipticrestrictedthree bodies under the radiating and oblate Primaries, Astrophysics and Space Science ,341, 349358.https://doi.org/10.1007/s10509-012-1109-3.

[16] Narayan a\&Usha T (2014), Stability of triangular equilibrium points in the elliptic restricted problem of three bodies with radiation and triaxial primaries, Astrophysics and Space Science, 351(1), 135-142.https://doi.org/10.1007/s10509-014-1818-x.

[17] UshaT, NarayanA\&Ishwar B (2014), Effects of radiation and triaxiality of primaries on triangular equilibrium points in elliptic restricted three body problem, Astrophysics and Space Science, 349(1), 151-164.https://doi.org/10.1007/s10509-013-1655-3.

[18] F aAbd El-Salam (2015), Stability of Triangular equilibrium points in the elliptic restricted three body problem with oblate and triaxial Primaries,

AstrophysSpaceSci, 357:15.https://doi.org/10.1007/s10509-015-2308-5.

[19] IshwarB \&Elipe A (2001), Secular Solutions at Triangular Equilibrium Point in the Generalized Photogravitational Restricted ThreeBody Problem, Astrophysics and Space Science, 277, No.3, $437-$ 446.https://doi.org/10.1023/A:1012528929233.

[20] Subbarao PV \& Sharma R K (1975), A Note on the Stability of the Triangular points of Equilibrium in the Restricted-Three body problem, Astronomy and Astrophysics, 43,381-383.

[21] KhannaM \&Bhatnagar K B (1998), Existence and Stability of the Libration points in the restricted three body problem when the smaller primary is a triaxial rigid body, Indian Journal of Pure and Applied Mathematics,29(10), 1011-1023. 